\title{
Single and Multiple Intrabeam Scattering in Hadron Colliders
}

\author{
Valeri Lebedev \\ FNAL, P.O. Box 500, Batavia, IL 60510, USA
}

\begin{abstract}
Single and multiple intra-beam scattering are usually considered separately. Such separation works well for electron-positron colliders but usually yields only coarse description in the case of hadron colliders. Boltzmann type integro-differential equation is used to describe evolution of longitudinal distribution due to IBS. The finite size of the longitudinal potential well, its non-linearity and $x-y$ coupling are taken into account. The model predictions for longitudinal and transverse distributions are compared to the experimental measurements.
\end{abstract}

\section{INTRODUCTION}

The intrabeam scattering (IBS) is closely related to the particle scattering in plasma and is well understood conceptually ${ }^{[1]}$. In the most general case IBS is described by Boltzmann type integro-differential equation but its exact solution is not easy even in simple cases. In this paper IBS is considered in application to high energy hadron colliders. Because of large $\gamma$, the longitudinal velocity spread is much smaller than the transverse in the beam frame resulting in a simplified description. First, we consider the emittance growth rates for Gaussian beams in the case of $x-y$ coupled motion. This approximation works well for transverse degrees of freedom; but due to longitudinal focusing nonlinearity and finite size of RF bucket it yields a coarse description of longitudinal distribution. To correct this problem we build the Fokker-Planck equation ${ }^{[2]}$ describing the longitudinal distribution. Then we generalize equations to include the single-scattering which dominates particle loss at the beginning of the process and is slowly diminishing with bunch lengthening. Finally, we compare the theory predictions with the observed beam parameter evolution during Tevatron stores.

\section{IBS RATES FOR COUPLED BEAM}

The evolution of distribution function for particle scattering in the plasma is determined by Landau collision integral ${ }^{[1]}$ :

$$
\frac{\partial f}{\partial t}=-\frac{\partial}{\partial p_{i}}\left(F_{i} f\right)+\frac{1}{2} \frac{\partial}{\partial p_{i}}\left(D_{i j} \frac{\partial f}{\partial p_{j}}\right) .
$$

Here the friction and diffusion are

$$
\begin{aligned}
& F_{i}(p)=-\frac{4 \pi n e^{4} L_{c}}{m} \int f\left(p^{\prime}\right) \frac{u_{i}}{|\mathbf{u}|^{3}} d^{3} p^{\prime}, \\
& D_{i j}=4 \pi n e^{4} L_{c} \int f\left(p^{\prime}\right) \frac{u^{2} \delta_{i j}-u_{i} u_{j}}{|\mathbf{u}|^{3}} d^{3} p^{\prime}, \\
& \mathbf{u}=\mathbf{v}-\mathbf{v}^{\prime},
\end{aligned}
$$

$n$ is the particle density, $e$ and $m$ are the particle charge and mass, and $L_{c}$ is the Coulomb logarithm. Multiplying Eq. (1) by $p_{x}^{2}$, and integrating it with Gaussian distribution,

$$
f=\frac{1}{(2 \pi)^{3 / 2} \sigma_{p x} \sigma_{p y} \sigma_{p z}} e^{-\frac{p_{x}{ }^{2}}{2 \sigma_{p x}^{2}-\frac{p_{y}{ }^{2}}{2 \sigma_{p y}^{2}}-\frac{p_{z}{ }^{2}}{2 \sigma_{p z}^{2}}},}
$$

one obtains

$$
\frac{d}{d t} \overline{\mathrm{p}_{x}{ }^{2}}=\frac{(2 \pi)^{3 / 2} n e^{4} m L \Psi\left(\sigma_{p x}, \sigma_{p y}, \sigma_{p z}\right)}{\sqrt{{\sigma_{p x}}^{2}+\sigma_{p y}{ }^{2}+\sigma_{p z}{ }^{2}}},
$$

where

$$
\begin{aligned}
& \Psi(x, y, z)=\frac{\sqrt{2}}{\pi} \sqrt{x^{2}+y^{2}+z^{2}} \\
& \int_{0}^{\infty} \frac{\left(\frac{y^{2}-x^{2}}{y^{2}+t^{2}}+\frac{z^{2}-x^{2}}{z^{2}+t^{2}}\right) t^{3} d t}{\left(x^{2}+t^{2}\right)^{3 / 2}\left(y^{2}+t^{2}\right)^{1 / 2}\left(z^{2}+t^{2}\right)^{1 / 2}} .
\end{aligned}
$$


The growth rates for two other degrees of freedom are obtained from Eq. (4) by cyclic substitution. For the case of pancake distribution it can be approximated as

$$
\Psi(0, x, y) \approx 1+\frac{\sqrt{2}}{\pi} \ln \left(\frac{x^{2}+y^{2}}{2 x y}\right)-.055\left(\frac{x^{2}-y^{2}}{x^{2}+y^{2}}\right)^{2} .
$$

This function possesses correct asymptotics and coincides with exact result within $\sim 1 \%$.

To describe IBS in a ring Eq. (4) needs to be averaged across the bunch. Below we limit ourselves to the case of pancake distribution, $\left(\Delta p_{\|} / p\right) / \gamma<<\theta_{\perp}$ and the energy well above the critical energy, $\gamma>>\gamma_{c r}$, which is typical for the high-energy colliders. Then, there is no significant difference between scattering in plasma and IBS for longitudinal degree of freedom. Averaging Eq. (4) over the Gaussian distribution in the $3 \mathrm{D}$ space and taking into account that the energy is equally distributed between potential and kinetic, yields the momentum spread growth rate ${ }^{[3]}$ :

$$
\frac{d}{d t}\left(\sigma_{p}^{2}\right)=\frac{e^{4}\left\langle\frac{N}{\sigma_{1} \sigma_{2} \sigma_{s}} \frac{\Psi\left(0, \theta_{1}, \theta_{2}\right)}{\sqrt{\theta_{1}^{2}+\theta_{2}^{2}}} L_{C}\right\rangle_{s}}{4 \sqrt{2} m^{2} c^{3} \gamma^{3} \beta^{3}} .
$$

Here $\sigma_{p} \equiv \sqrt{\overline{(\Delta p / p)^{2}}}$ is the rms momentum spread, $\sigma_{1}, \sigma_{2}, \theta_{1}$ and $\theta_{2}$ are the rms ellipse semi-axis in the $x-y$ plane and in the plane of local angular spreads $\left(\theta_{x}-\right.$ $\left.\theta_{y}\right), N$ is the number of particles in the bunch, and index $s$ denotes averaging over the ring circumference. To find the ellipse sizes we describe the $x-y$ coupled betatron motion using an extension of the Mais-Ripken representation $^{[4]}$ :

$$
\hat{\mathbf{x}}(s)=\operatorname{Re}\left(\widetilde{\varepsilon}_{1} \mathbf{v}_{1}(s) e^{-i \mu_{1}(s)}+\widetilde{\varepsilon}_{2} \mathbf{v}_{2}(s) e^{-i \mu_{2}(s)}\right),
$$

where $\mathbf{x}(\mathrm{s})=\left[x, \theta_{\mathrm{x}}, y, \theta_{\mathrm{y}}\right]^{T}$ is the coordinate vector, $\mathbf{v}_{1}$ and $\mathbf{v}_{2}$ are the eigen-vectors,

$$
\mathbf{v}_{1}=\left[\begin{array}{c}
\sqrt{\beta_{1 x}} \\
-\frac{i(1-u)+\alpha_{1 x}}{\sqrt{\beta_{1 x}}} \\
\sqrt{\beta_{1 y}} e^{i v_{1}} \\
i u+\alpha_{1 y} \\
-\frac{\sqrt{\beta_{1 y}}}{2} e^{i v_{1}}
\end{array}\right], \quad \mathbf{v}_{2}=\left[\begin{array}{c}
\sqrt{\beta_{2 x}} e^{i v_{2}} \\
-\frac{i u+\alpha_{2 x}}{\sqrt{\beta_{2 x}}} e^{i v_{2}} \\
\sqrt{\beta_{2 y}} \\
-\frac{i(1-u)+\alpha_{2 y}}{\sqrt{\beta_{2 y}}}
\end{array}\right],
$$

$\beta$ 's and $\alpha$ 's represent the extension of the beta- and alpha-functions for uncoupled motion, and the parameters $u, v_{1,2}$ are determined by symplecticity condition from $\beta$ 's and $\alpha$ 's. The bilinear form inscribing particle ellipsoid in 4D phase space,

$$
\hat{\mathbf{x}}^{T} \Xi \hat{\mathbf{x}}=1,
$$

can be expressed through the eigen-vectors and the emittances. Corresponding formulas can be found in Ref. [4]. To obtain the beam sizes and angular spreads we also need to take into account the dispersion contribution into the beam sizes and angular spreads. In this case the distribution function is:

$$
f\left(\hat{\mathbf{x}}, \theta_{\|}\right) \propto \exp \left(-\frac{\left(\hat{\mathbf{x}}-\mathbf{D} \theta_{\|}\right)^{T} \Xi\left(\hat{\mathbf{x}}-\mathbf{D} \theta_{\|}\right)}{2}-\frac{\theta_{\|}^{2}}{2 \sigma_{p}^{2}}\right),(1
$$

where $\mathbf{D}=\left[D_{x}, D_{x}^{\prime}, D_{y}, D_{y}^{\prime}\right]^{T}$ is the vector of dispersions. Integrating over momentum spread we obtain 4D Gaussian distribution

$f(\hat{\mathbf{x}}) \propto \exp \left(-\frac{\hat{\mathbf{x}}^{T} \Xi^{\prime} \hat{\mathbf{x}}}{2}\right), \quad \Xi^{\prime}=\Xi-\frac{\Xi \mathbf{D D}^{T} \Xi}{\sigma_{p}^{-2}+\mathbf{D}^{T} \Xi \mathbf{D}}$.

That yields the beam sizes and local angular spreads:

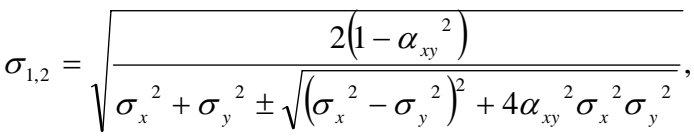

$$
\begin{aligned}
& \theta_{1,2}=\sqrt{\frac{2}{\Xi_{22}^{\prime}+\Xi_{44}^{\prime} \pm \sqrt{\left(\Xi_{22}^{\prime}-\Xi_{44}^{\prime}\right)^{2}+4 \Xi_{24}^{\prime 2}}}}, \\
& \sigma_{x}=\sqrt{\varepsilon_{1} \beta_{1 x}+\varepsilon_{2} \beta_{2 x}+D_{x}{ }^{2} \sigma_{p}{ }^{2}} \text {, } \\
& \sigma_{y}=\sqrt{\varepsilon_{1} \beta_{1 y}+\varepsilon_{2} \beta_{2 y}+D_{y}{ }^{2} \sigma_{p}{ }^{2}} \text {, } \\
& \alpha_{x y}=\frac{\varepsilon_{1} \sqrt{\beta_{1 x} \beta_{1 y}} \cos v_{1}+\varepsilon_{2} \sqrt{\beta_{2 x} \beta_{2 y}} \cos \nu_{2}+D_{x} D_{y} \sigma_{p}{ }^{2}}{\sigma_{x} \sigma_{y}}
\end{aligned}
$$

For $\gamma>\gamma_{c r}$, the main source of the transverse emittance growth is related to the excitation of betatron oscillations at regions with non-zero dispersion. In this case the momentum change and the amplitudes of particle motion are related as,

$$
\mathbf{D} \frac{\Delta p}{p}=\operatorname{Re}\left(a_{1} \mathbf{v}_{1}+a_{2} \mathbf{v}_{2}\right) \equiv \mathbf{V a},
$$

where $\mathbf{V}=\left[\mathbf{v}_{1}^{\prime},-\mathbf{v}^{\prime \prime}{ }_{1}, \mathbf{v}_{2}^{\prime},-\mathbf{v}^{\prime \prime}{ }_{2}\right], \mathbf{a}=\left[\mathrm{a}_{1}^{\prime}, \mathrm{a}^{\prime \prime}{ }_{1}, \mathrm{a}_{2}{ }_{2}, \mathrm{a}_{2}{ }_{2}\right]$. Expressing emittances through coordinates of vector $\mathbf{a}$,

$$
\mathbf{a}=\frac{\Delta p}{p} \mathbf{V}^{-1} \mathbf{D}
$$

and averaging yield the emittance growths rates for two transverse betatron modes:

$$
\begin{aligned}
& \frac{d \varepsilon_{1,2}}{d t}=\left\langle A_{1,2} \frac{d}{d t} \overline{\left(\frac{\Delta p}{p}\right)^{2}}\right\rangle, \\
& A_{1,2}=\mathbf{D}^{T} \mathbf{B}_{1,2} \mathbf{D}, \quad \mathbf{B}_{1}=\left(\mathbf{V}^{-1}\right)^{T}\left[\begin{array}{ll}
\mathbf{I} & \mathbf{0} \\
\mathbf{0} & \mathbf{0}
\end{array}\right] \mathbf{V}^{-1}, \\
& \mathbf{B}_{2}=\left(\mathbf{V}^{-1}\right)^{T}\left[\begin{array}{ll}
\mathbf{0} & \mathbf{0} \\
\mathbf{0} & \mathbf{I}
\end{array}\right] \mathbf{V}^{-1}, \quad \mathbf{I}=\left[\begin{array}{ll}
1 & 0 \\
0 & 1
\end{array}\right] .
\end{aligned}
$$

For uncoupled case Eqs. (7) and (17) coincide with results presented in Ref. [3]. 


\section{IBS IN NONLINEAR WELL}

In the case of non-linear oscillator one needs to make a transformation to the angle-phase variables and to perform averaging over phase. For $\mathrm{v}_{\|}<<\mathrm{v}_{\perp}$ one can neglect friction in Eq. (1) that results in:

$$
\frac{\partial f}{\partial t}=\frac{1}{2} \frac{\partial}{\partial I}\left(I \frac{D(I)}{\omega(I)} \frac{\partial f}{\partial I}\right) .
$$

Here $\omega$ and $I$ are the frequency and action for the dimensionless Hamiltonian,

$$
H=\frac{p^{2}}{2}+2\left(\sin \frac{\varphi}{2}\right)^{2} .
$$

The diffusion coefficient is determined as:

$$
\begin{aligned}
& D(I)=4 L_{c} \tilde{A}(\oint n(\varphi) p d \varphi / \oint p d \varphi), \\
& \tilde{A}=\sqrt{\frac{\pi}{2}} \frac{\pi^{2} \alpha N e^{4} q^{2}}{e V_{0} m c \beta \gamma^{2} C}\left\langle\frac{1}{\sigma_{1} \sigma_{2}} \frac{\Psi\left(0, \theta_{1}, \theta_{2}\right)}{\sqrt{\theta_{1}^{2}+\theta_{2}^{2}}}\right\rangle_{s},
\end{aligned}
$$

where $C$ is the ring circumference, $\alpha$ is the momentum compaction, $q$ is the harmonic number, and $V_{0}$ is the RF voltage, $n(\varphi)$ is the linear density $\int_{-\pi}^{\pi} n(\varphi) d \varphi=1$.

To take into account single scattering we follow the method developed in Ref. [5]. For high energy collider the maximum longitudinal velocity determined by the $\mathrm{RF}$ bucket size is much smaller than the transverse ones, $\theta_{\| \max } / \gamma<<\theta_{\perp}$, and one can write:

$$
\begin{aligned}
& \frac{\partial f}{\partial t}=\left\langle\tilde{A} \int n(\varphi) \frac{f(p+q)-f(p)}{|q|^{3}} \mathrm{~d} q\right\rangle_{\text {period }}= \\
& \left\langle\tilde{A} \int n(\varphi) \frac{f\left(I^{\prime}\right)-f(I)}{\left|p-p^{\prime}\right|^{3}} \delta\left(\varphi-\varphi^{\prime}\right) \mathrm{d} I^{\prime} d \psi d \psi^{\prime}\right\rangle_{\text {period }},
\end{aligned}
$$

which after averaging yields:

$$
\frac{\partial f(I, t)}{\partial t}=\tilde{A} \int_{0}^{\infty} W\left(I, I^{\prime}\right)\left(f\left(I^{\prime}, t\right)-f(I, t)\right) \mathrm{d} I^{\prime} .
$$

The kernel is a symmetrical function, $W\left(I, I^{\prime}\right)=W\left(I^{\prime}, I\right)$, which for $I^{\prime} \geq I$ can be expressed as following

$$
\begin{aligned}
& W\left(I, I^{\prime}\right)=\frac{\omega \omega^{\prime}}{\pi\left(H(I)-H\left(I^{\prime}\right)\right)^{3}} \\
& \quad\left[\left(H(I)-H\left(I^{\prime}\right)\right) \int_{0}^{a(I)} n(\varphi) \frac{d \varphi}{p}+2 \int_{0}^{a(I)} n(\varphi) p d \varphi\right] .
\end{aligned}
$$

The kernel divergence at $I=I^{\prime}$ needs to be limited at the minimum action difference corresponding to the maximum impact parameter usually determined by the beam size. Numerical solution of Eq. (24) is carried out similar to Ref. [5].

\section{DISCUSSION}

To achieve good agreement between the theory and the experiments it was critical to include other beamloss and diffusion mechanisms: the elastic and nonelastic scattering on the residual gas, particle loss due luminosity, and the longitudinal diffusion due to RF noise. Figure 1 presents the measured and computed dependencies of proton and antiproton bunch lengths during one of Tevatron stores. Similar good coincidence has been obtained for the horizontal and vertical beam emittances. Measured Tevatron optics with $x$-y coupling taken into account has been used in the calculations. The saturation of antiproton bunch lengthening in the second half of the store is related to the beam-beam effects, which make transverse motion unstable for particles with large synchrotron amplitudes. At $t=28$ hour a corrector power supply was lost. That worsened beam-beam effects with consecutive growth of particle loss and nullifying bunch lengthening.

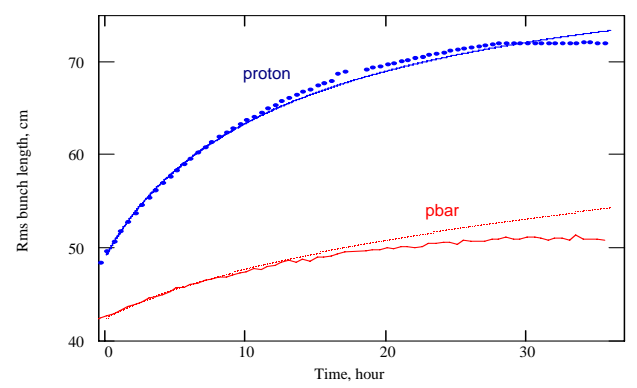

FIGURE 1. Measured (dotted line) and computed (solid line) dependence of rms bunch length on time for Store 3678.

\section{ACKNOWLEDGMENTS}

The author thanks A. Burov for fruitful discussions and A. Tollestrup for help in data analysis.

\section{REFERENCES}

1. Landau, L. D. Phys. Zs. Sowjet, 10, p. 154, 1936.

2. Wei, J. and Ruggiero, A.G., Proc. 1991 Part. Accelerator Conference, San-Francisco, pp. 1871-1873.

3. Bjorken, J. and Mtingwa, S., Part. Accel. 13, p. 115 (1983).

4. Lebedev, V. and Bogacz, S., "Betatron motion with coupling of horizontal and vertical degrees of freedom", JLAB-ACC-99-19, http://www.jlab.org/div_dept/admin/ publications/papers/99/ACC99-19.pdf, 1999.

5. Lebedev, V. and Nagaitsev, S., Proc. 2002 European Part. Accelerator Conference, Paris, pp. 1362-1364. 